\title{
Minimally invasive distal pancreatectomy: a case-matched cost-analysis between robot-assisted surgery and direct manual laparoscopy
}

\author{
Gregorio Di Franco ${ }^{1,5} \cdot$ Andrea Peri $^{2} \cdot$ Valentina Lorenzoni $^{3} \cdot$ Matteo Palmeri ${ }^{1,5} \cdot$ Niccolò Furbetta $^{1,5}$. \\ Simone Guadagni ${ }^{1,5}$. Desirée Gianardi ${ }^{1,5}$. Matteo Bianchini ${ }^{1,5}$. Luca Emanuele Pollina ${ }^{4}$. Franca Melfi ${ }^{5}$. \\ Domenica Mamone $^{6} \cdot$ Carlo Milli $^{7}$. Giulio Di Candio ${ }^{1} \cdot$ Giuseppe Turchetti $^{3} \cdot$ Andrea Pietrabissa $^{2} \cdot$ Luca Morelli $^{1,5,8} \mathbb{C}_{0}$
}

Received: 12 October 2020 / Accepted: 13 January 2021 / Published online: 3 February 2021

(c) The Author(s) 2021

\begin{abstract}
Background Few studies have reported a structured cost analysis of robotic distal pancreatectomy (RDP), and none have compared the relative costs between the robotic-assisted surgery (RAS) and the direct manual laparoscopy (DML) in this setting. The aim of the present study is to address this issue by comparing surgical outcomes and costs of RDP and laparoscopic distal pancreatectomies (LDP).

Methods Eighty-eight RDP and 47 LDP performed between January 2008 and January 2020 were retrospectively analyzed. Three comparable groups of 35 patients each (Si-RDP-group, Xi-RDP group, LDP-group) were obtained matching 1:1 the RDP-groups with the LDP-group. Overall costs, including overall variable costs (OVC) and fixed costs were compared using generalized linear regression model adjusting for covariates.

Results The conversion rate was significantly lower in the Si-RDP-group and Xi-RDP-group: $2.9 \%$ and 0\%, respectively, versus $14.3 \%$ in the LDP-group $(p=0.045)$. Although not statistically significant, the mean operative time was lower in Xi-RDP-group: $226 \mathrm{~min}$ versus $262 \mathrm{~min}$ for Si-RDP-group and $247 \mathrm{~min}$ for LDP-group. The overall post-operative complications rate and the length of hospital stay (LOS) were not significantly different between the three groups. In LDP-group, the LOS of converted cases was significantly longer: 15.6 versus 9.8 days $(p=0.039)$. Overall costs of LDP-group were significantly lower than RDP-groups, $(p<0.001)$. At multivariate analysis OVC resulted no longer statistically significantly different between LDP-group and Xi-RDP-group $(p=0.099)$, and between LDP-group and the RDP-groups when the spleen preservation was indicated ( $p=0.115$ and $p=0.261$ for Si-RDP-group and Xi-RDP-group, respectively).

Conclusions RAS is more expensive than DML for DP because of higher acquisition and maintenance costs. The flattening of these differences considering only the variable costs, in a high-volume multidisciplinary center for RAS, suggests a possible optimization of the costs in this setting. RAS might be particularly indicated for minimally invasive DP when the spleen preservation is scheduled.
\end{abstract}

Keywords Robotic distal pancreatectomy $\cdot$ Laparoscopic distal pancreatectomy $\cdot$ Costs' analysis

Luca Morelli

luca.morelli@unipi.it

1 General Surgery Unit, Department of Translational Research and New Technologies in Medicine and Surgery, University of Pisa, Via Paradisa 2, 56125 Pisa, Italy

2 Department of Surgery, Fondazione IRCCS Policlinico San Matteo, Pavia, Italy

3 Institute of Management, Scuola Superiore Sant'Anna, Pisa, Italy
4 Second Division of Surgical Pathology, University Hospital of Pisa, Pisa, Italy

5 Multidisciplinary Center of Robotic Surgery, University Hospital of Pisa, Pisa, Italy

6 Pharmaceutical Unit, Medical Device Management, University Hospital of Pisa, Pisa, Italy

Board of Directors, University Hospital of Pisa, Pisa, Italy

8 EndoCAS (Center for Computer Assisted Surgery), University of Pisa, Pisa, Italy 
Since the description of the first minimally invasive distal pancreatectomy (MIDP) in 1994 [1], the use of the laparoscopic technique for distal pancreatectomy has progressively increased. In fact, MIDP is now considered the standard approach for benign and premalignant pancreatic tumors, especially in centers with high experience with pancreatic surgery [2]. Moreover, also for malignant pancreatic tumors, the MIDP seems to be equivalent to the traditional open approach in terms of oncological outcomes (i.e., R0 resection, resection margins, harvested lymph nodes, 30-day mortality, disease-free survival, and overall survival) [2]. However, although MIDP has been associated to clear advantages over the open approach in terms of pain, intraoperative blood loss, and length of hospital stay (LOS) [3-5], it is still performed with a traditional approach in most hospitals, because of the deep anatomical location of the pancreas and the complexity of distal pancreatectomy. In this regard, the widespread adoption of robotic-assisted surgery (RAS) also in pancreatic surgery could favor the increasing of MIDP.

Robotic distal pancreatectomy (RDP) seems also to be associated with an additional positive impact on the clinical outcomes as well, with a reduction of conversion rates, higher spleen preservation rates, and a shorter LOS $[2,6,7]$. A potential barrier to the diffusion of the robotic approach could be its economic impact [8]; indeed, it is well known that RAS is generally associated with higher costs in comparison to direct manual laparoscopy (DML), also for RDP, as data emerged from previous studies, although controversial, are almost unanimously oriented toward higher cost for RAS [3, 9-14]. However, despite at the beginning of the experience with the robotic platforms the economic analyses were strongly against the use of the da Vinci Surgical System, more recent data seem to have opened new perspectives in this regard [15]. Therefore, the aim of our study is to perform a structured cost-analysis, including the shortterm outcomes, of distal pancreatectomy performed with RAS, with both the da Vinci Si and Xi in a multidisciplinary robotic center, and with DML.

\section{Materials and methods}

From January 2008 to January 2020, 88 patients underwent RDP at the Multidisciplinary Center of Robotic Surgery of Pisa, performed by the 1st General Surgery Unit, and were included in the present study. All patients were operated by two surgeons (AP and LM), both with high experience of pancreatic surgery ( $>300$ procedures each) and minimally invasive surgery (both laparoscopic and robotic surgery, $>300$ procedures each), and both trained in the highvolume center of Pisa (> 100 pancreatic resections/year).

The choice of the operative technique (laparoscopic or robot-assisted) was at the discretion of the operating surgeon and based on the robotic platform availability, being that the second one has been the preferred approach in the last 8 years.

The RDPs were performed with the da Vinci Si platform until December 2014. Since the introduction of the da Vinci $\mathrm{Xi}$ at our center, in January 2015, the choice of the platform of da Vinci Surgical System (da Vinci Si or da Vinci Xi) depended on their availability. In detail, 47 patients underwent RDP with the da Vinci Si (Si-RDP-group) and 41 patients with the da Vinci Xi (Xi-RDP-group). In the same period 47 patients underwent laparoscopic distal pancreatectomy (LDP-group) for the same indications by the same two surgeons (AP and LM). In order to compare outcomes and costs among the three groups minimizing possible biases deriving from treatment allocation, three comparable groups were obtained matching 1:1 the two robotic groups with the LDP-group. The following patients' characteristics were considered for the matching: age, gender, Body Mass Index (BMI), and American Society of Anesthesiology (ASA) score. After matching, the final population included 105 patients, equally divided between each group: Si-RDP-group ( $n=35)$, Xi-RDP group $(n=35)$, and LDP-group $(n=35)$.

Data on patients' preoperative characteristics, surgical procedures, post-operative course, follow-up and resources used (i.e., associated to operative time, length of stay, etc.) were retrospectively reviewed and analyzed, from a prospectively collected database.

The preoperative workup included abdominal ultrasonography, abdomen CT and/or MRI. Indications for minimally invasive approach were neoplasms $<10 \mathrm{~cm}$ with benign or borderline features on cross-sectional imaging or adenocarcinomas, similarly to other teams $[11,16]$.

\section{Surgical procedures}

Laparoscopic and robotic distal pancreatectomies were performed as already described [17]. Splenic preservation was performed only in case of presumed benign or premalignant lesions and pursued according to the splenic vessels conservation technique described by Kimura et al. [18]. Splenic conservation performed by sacrificing the splenic vessels (Warshaw technique [19]) was not performed in our series. In both laparoscopic or robotic techniques, parenchymal transection and closure was carried out using electrocautery and the stump was over sewn with intracorporeal suturing and knotting, or with an endostapler.

Laparoscopic DP The patient is placed in the supine or left sided position, dependent upon the tumor site, with both arms along the sides of the body, and tilted in partial reverse Trendelenburg position. Four/five ports are used (2/3, $5 \mathrm{~mm}$; $2,12 \mathrm{~mm}$ ). After the establishment of pneumoperitoneum and the ports placement, an abdominal exploration is performed. The lesser sac is then entered through the greater 
gastrocolic omentum. The splenic flexure of the colon is mobilized, if necessary. An intra-operative diagnostic ultrasound with a laparoscopic probe is always performed to evaluate the pancreatic lesion and its correct position. The superior and inferior borders of the pancreas are defined and the pancreas is transected using a stapler (ETS Flex 45 Endoscopic Articulating Staple, Johnson \& Johnson, USA). The distal transected pancreas is gently lifted, and a medialto- lateral dissection is started. In case of splenic conservation, the splenic vein and artery are skeletonized from the isthmus toward the splenic hilum. This manoeuvre allows both the lymphadenectomy and a step-by-step division of all the branches coming from the splenic vessels. In case of DP with splenectomy, first the splenic artery and then the vein are divided after the transection of the pancreas. This step is followed by a medial-to-lateral dissection posterior to the splenic vein along the retroperitoneal plane. Two drains are left near the pancreatic stump.

Robotic DP Patient position is similar to that of laparoscopic DP. For this approach, a five-port technique is adopted. The trocars disposition and the robot chart position change according to the type of robot used (Figs. 1 and 2). For dissection and retraction, monopolar scissor and
Cadiere grasper are used, while the robotic energy devices employed are Gyrus PK SuperPulse Generator (Olympus, Center Valley, PA, USA) with the da Vinci Si or Maryland bipolar forceps (Intuitive Surgical, Sunnyvale, California, USA) during the last cases of the da Vinci Si or with the da Vinci Xi. The assistant's trocar is then inserted and used for sutures insertion or suction. The surgical steps are similar to those of the laparoscopic approach. The pancreas is divided with robotic monopolar curved scissors and then the body of the pancreas is pulled up with the fourth arm to expose the posterior attachments of the organ. The special pulsemodulating robotic device (PKTM) or the Maryland bipolar forceps are used to seal all small tributary splenic vessels. The remnant pancreatic stump is predominantly oversewn with 4 or 5 interrupted sutures using robotic needle drivers with selective ligation of Wirsung duct. In case of thickness of the pancreas, the parenchyma is instead transected with a laparoscopic endostapler or with a robotic EndoWrist stapler for the da Vinci Xi. Once the gland is divided and fully freed from the attachments, the robot is undocked, and the specimen is placed in a plastic bag for a laparoscopic extraction through a suprapubic incision. Two drains are left near the pancreatic stump.
Fig. 1 Trocars' disposition and robotic instruments used for RDP with the da Vinci Si
Fig. 2 Trocars' disposition and robotic instruments used for RDP with the da Vinci Xi
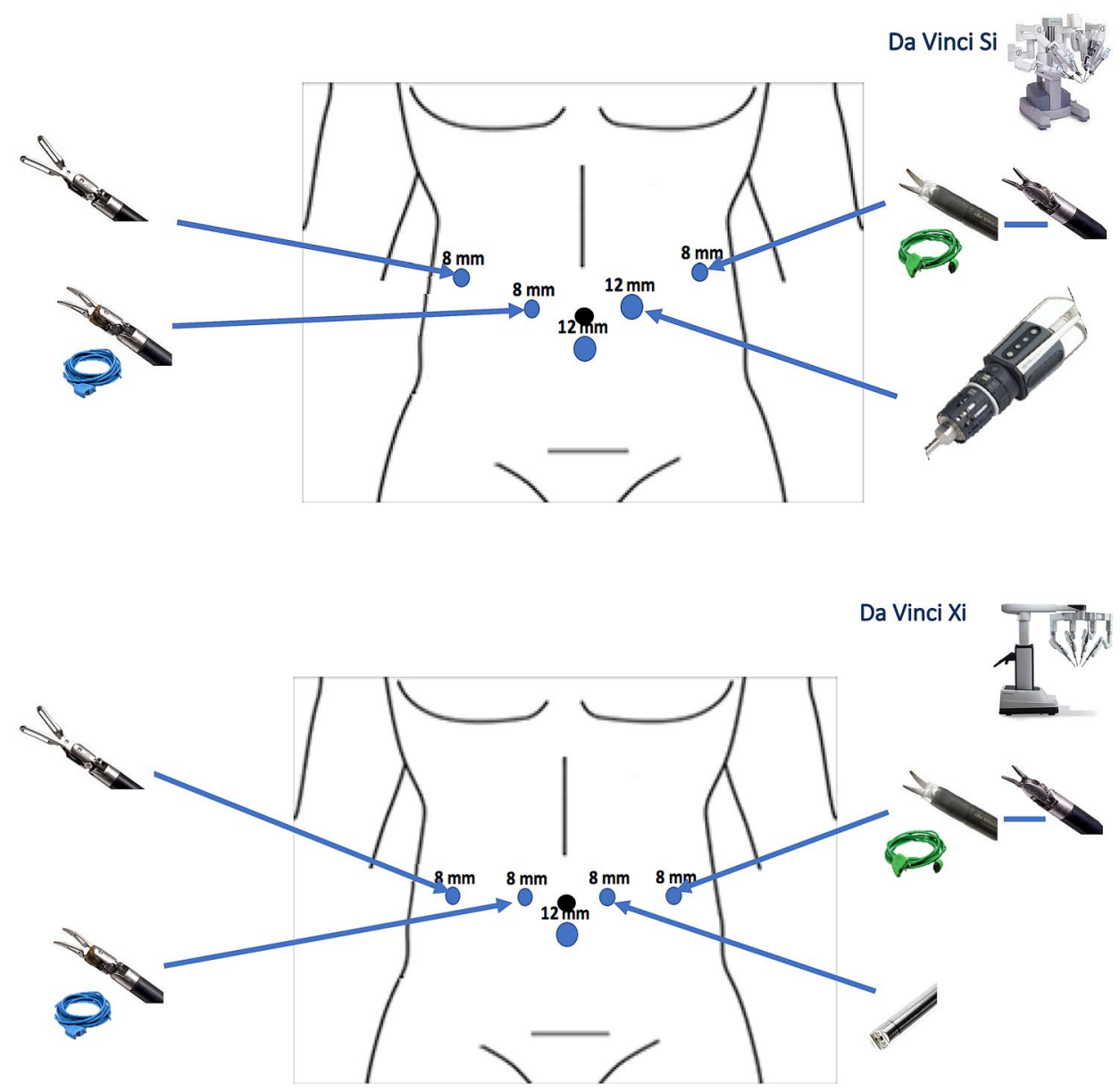


\section{Data collection}

Preoperative data included age, gender, body mass index (BMI), American Society of Anesthesiology (ASA) score, preoperative diagnosis, intention to spleen preservation, and radiologic tumor size. Operative data included type of surgical procedure, operative time (OT), conversion rate, spleen preservation rate (calculated in those cases in which the spleen preservation was scheduled preoperatively on the basis of preoperative diagnosis), any additional organ resection (beyond splenectomy), pancreatic stump management (handsewn or stapler and number of charges used), and intraoperative complications. Postoperative data included LOS, both considering intensive care unit (ICU) and general ward, complications (according to the Clavien-Dindo Classification [20]), re-operation rate, and mortality. The surgical complications comprised surgical site infections, post-pancreatectomy hemorrhage [21], delayed gastric emptying [22], and post-operative pancreatic fistula (POPF) [23]. Pathological data included the final histology, tumor size, and the number of harvested lymph nodes.

\section{Costs analysis}

Costs were valued using a micro-costing approach and expressed in euros and referred to 2020. Unit costs were collected from the accounting department of the hospital and divided in fixed costs and variable costs. In detail, fixed costs included the purchase and maintenance costs of the technology and the proportion of fixed costs attributable to a single intervention was estimated firstly deriving a cost per year on the basis of acquisition costs, the amortization period and annual maintenance costs, then dividing these costs for the overall number of interventions for which each technology was used. Overall variable costs (OVC) included items related to disposable instruments used within each intervention (consumable costs, $\mathrm{CCs}$ ), operating room personnel (personnel costs, PCs), and length of stay, both ICU and general ward (hospital stay costs, HCs). For each intervention variable costs associated with the specific intervention were then estimated valuing resources used according to unit costs collected. Details about resources use and related costs are reported in Tables 1, 2, 3, 4 and 5.

Robotic staplers' costs were excluded from the consumable cost analysis to avoid bias, as they were introduced only since 2016, and the choice to use them just depends on surgeon's preference. In order to have three homogeneous groups, when the robotic staplers were used in the $\mathrm{Xi}$ RDP-group, we considered the costs of the corresponding laparoscopic staplers and charges.

The study was approved by the Institutional review board.

Table 1 Details of fixed costs

\begin{tabular}{llll}
\hline & LDP & Si-RDP & Xi-RDP \\
\hline Fixed costs attributable to the single intervention ${ }^{*}(€)$ & 34.41 & 1583.75 & 1827.25 \\
\hline
\end{tabular}

$L D P$ laparoscopic distal pancreatectomy, Si-RDP robotic distal pancreatectomy with da Vinci Si, Xi-RDP robotic distal pancreatectomy with da Vinci Xi

*The costs are estimated dividing the acquisition costs for the amortization period, then considering annual maintenance costs and finally dividing annual costs by the overall number of interventions performed with the specific technology

Table 2 Personnel and hospital stay cost for MIDP

\begin{tabular}{llc}
\hline Personnel group & Unit cost $(€ / \mathrm{h})$ & $\begin{array}{c}\text { Number of } \\
\text { figures }\end{array}$ \\
\hline Surgeon & 62.69 & 2 \\
Anesthetist & 65.63 & 1 \\
Anesthesiology technologist & 27.96 & 1 \\
Surgical nurse & 30.50 & 1 \\
Surgical technologist & 31.34 & 1 \\
Nurse assistant & 21.36 & 1 \\
\hline & Unit cost $(€ / \mathrm{h})$ & - \\
\hline Hospital stay costs in a surgical ward & 420.00 & - \\
Hospital stay costs in an ICU & 1150.00 & \\
\hline
\end{tabular}

ICU intensive care unit 
Table 3 Consumable cost of LDP-group

\begin{tabular}{lrll}
\hline & Unit costs $(€)$ & Quantity & Overall CC $(€)$ \\
\hline 5 mm laparoscopic trocar & 36.60 & 1 & 36.60 \\
12 mm laparoscopic trocar & 48.00 & 1 & 48.00 \\
Echelon 60 & 334.00 & 1 & 334.00 \\
Echelon 45 & 298.00 & 1 & 298.00 \\
Echelon 60 stapler charge & 176.00 & $\#$ & $\#$ \\
Echelon 45 stapler charge & 190.00 & $\#$ & $\#$ \\
Prolene suture & 7.00 & 5 & 35.00 \\
Verees needle & 6.13 & 1 & 6.13 \\
Ultracision & 640.00 & 1 & 640.00 \\
\hline
\end{tabular}

$C C$ consumable cost

${ }^{\#}$ On the basis of the number of laparoscopic charges used in each case

Table 4 Consumable cost of Si-RDP-group

\begin{tabular}{lcll}
\hline & Unit costs $(€)$ & Quantity & Overall CC $(€)$ \\
\hline Instrument arm drape & 128.93 & 1 & 128.93 \\
Camera arm drape & 120.00 & 1 & 120.00 \\
Camera head drape & 117.02 & 1 & 117.02 \\
Cadiere forceps & 568.57 & 1 & 568.57 \\
Maryland bipolar forceps & 769.00 & 1 & 769.00 \\
Monopolar curved scissors & 909.73 & 1 & 909.73 \\
Tip cover accessory & 57.52 & 1 & 57.52 \\
Cannula seal 8 mm & 44.63 & 3 & 133.89 \\
8 mm bladeless obturator & 72.39 & 1 & 72.39 \\
12 mm laparoscopic trocar & 48.00 & 2 & 96.00 \\
Robotic large needle driver & 623.91 & $1^{*}$ & 623.91 \\
Prolene suture & 7.00 & 5 & 35.00 \\
Laparoscopic stapler & 219.00 & $1^{\circ}$ & 219.00 \\
Laparoscopic stapler & 189.00 & $\#$ & $\#$ \\
$\quad$ charge & & & \\
Verees needle & 6.13 & 1 & 6.13 \\
Gelport & 353.00 & $1^{\S}$ & 353.00 \\
\hline
\end{tabular}

CC consumable cost

*In case of handsewn closure of pancreatic stump

${ }^{\circ}$ In case of section of the pancreas with a stapler

\# On the basis of the number of laparoscopic charges used in each case

${ }^{\S}$ In case of conversion to Hand-Assisted Laparoscopic Surgery (HALS procedure)

\section{Statistical analysis}

Categorical variables were depicted as number of cases and percentages, while continuous variables were expressed as mean \pm (standard deviation) or median [25-75 percentile], depending on their distribution. Chi-square test and Fisher test were used to compare the distribution of categorical variables. For continuous variables, comparisons were made using independent $T$-test or Mann-Whitney test and multiple
Table 5 Consumable cost of Xi-RDP-group

\begin{tabular}{lclc}
\hline & Unit costs $(€)$ & Quantity & Overall CC $(€)$ \\
\hline Instrument arm drape & 128.93 & 1 & 128.93 \\
Column drape & 61.53 & 1 & 61.53 \\
Cadiere forceps & 568.57 & 1 & 568.57 \\
Maryland bipolar forceps & 769.00 & 1 & 769.00 \\
Monopolar curved scis- & 909.73 & 1 & 909.73 \\
$\quad$ sors & & & \\
Tip cover accessory & 57.52 & 1 & 57.52 \\
Cannula seal 5-8 mm & 44.63 & 4 & 178.52 \\
8 mm bladeless obturator & 72.39 & 1 & 72.39 \\
12 mm laparoscopic & 48.00 & 1 & 48.00 \\
$\quad$ trocar & & & \\
Robotic large needle & 623.91 & $1 *$ & 623.91 \\
$\quad$ driver & & & \\
Prolene suture & 7.00 & 5 & 35.00 \\
Laparoscopic stapler & 219.00 & $1^{\circ}$ & 219.00 \\
Laparoscopic stapler & 189.00 & $\#$ & 189.00 \\
$\quad$ charge & & & \\
Verees needle & 6.13 & 1 & 6.13 \\
\hline
\end{tabular}

$C C$ consumable cost

*In case of handsewn closure of pancreatic stump

${ }^{\circ}$ In case of section of the pancreas with a stapler

${ }^{\#}$ On the basis of the number of laparoscopic charges used in each case

comparisons were performed by means of ANalysis Of Variance (ANOVA) or Kruskall-Wallis test. Post hoc comparisons were performed using Bonferroni or Mann-Whitney test with Bonferroni correction for p-value as appropriate.

Generalized linear models were used to evaluate the impact of the different surgical techniques on costs. In details, in addition to univariate analysis, multivariate models were developed including those variables whose $p$ value $<0.10$ at univariate analysis. In the multivariate analysis patients' age was forced to enter while variables creating collinearity (on the basis of the variance inflation factor) were excluded. A $p$-value $<0.05$ was considered statistically significant. All analyses were performed using Stata version 14.

\section{Results}

Preoperative data are summarized in Table 6. No differences were reported in the demographical data. No statistically significant differences were reported in the preoperative diagnoses between the three groups, even if a higher percentage of cystic lesions was reported in the Si-RDP-group (62.9\% in the Si-RDP vs $42.9 \%$ in the LDP-group and $34.3 \%$ in the $\mathrm{Xi}$-RDP-group), while a higher percentage of pancreatic carcinoma was reported in the LDP-group and Xi-RDP-group 
Table 6 Preoperative data

\begin{tabular}{|c|c|c|c|c|}
\hline & LPD-group & Si-RDP-group & Xi-RDP-group & $p$ value \\
\hline Age (years), mean $\pm S D$ & $63.9 \pm 16.9$ & $60.4 \pm 13.2$ & $60.3 \pm 14.5$ & 0.527 \\
\hline Male:Female (\%) & $17: 18(48.6: 51.4)$ & $11: 24(31.4: 68.6)$ & $18: 17(51.4: 48.6)$ & 0.189 \\
\hline BMI $\left(\mathrm{Kg} / \mathrm{m}^{2}\right)$, mean $\pm \mathrm{SD}$ & $26.0 \pm 5.5$ & $26.2 \pm 4.7$ & $26.0 \pm 4.4$ & 0.988 \\
\hline ASA score, $n(\%)$ & & & & 0.927 \\
\hline ASA I & $2(5.7)$ & $1(2.9)$ & $2(5.7)$ & \\
\hline ASA II & $19(54.3)$ & 17 (48.6) & $18(51.45)$ & \\
\hline ASA III & $14(40.0)$ & $17(48.6)$ & $14(40.0)$ & \\
\hline ASA IV & 0 & 0 & $1(2.9)$ & \\
\hline Preoperative diagnosis, $n(\%)$ & & & & 0.157 \\
\hline Pancreatic neoplasia & $14(40)$ & $6(17.1)$ & $13(37.1)$ & \\
\hline Cystic lesion & $15(42.9)$ & $22(62.9)$ & $12(34.3)$ & \\
\hline Neuroendocrine neoplasia & $6(17.1)$ & $7(20.0)$ & $9(25.7)$ & \\
\hline $\begin{array}{l}\text { Renal cell carcinoma pancre- } \\
\text { atic metastasis }\end{array}$ & 0 & 0 & $1(2.9)$ & \\
\hline Dimension $(\mathrm{cm})$, mean $\pm \mathrm{SD}$ & $4.0 \pm 2.2$ & $3.1 \pm 2.0$ & $3.5 \pm 1.5$ & 0.171 \\
\hline
\end{tabular}

$B M I$ body mass index, ASA score American society of anesthesiologists score

(40\% in the LDP-group and $37.1 \%$ in the Xi-RDP-group vs $17.1 \%$ in the Si-RDP-group).

Perioperative data are summarized in Table 7. Although not statistically significant, the mean operative time was slightly lower in Xi-RDP-group (225 min) with respect to the Si-RDP-group (262 $\mathrm{min}$ ), and to the LDP-group
(247 $\min ),(p=0.155)$. Regarding the intraoperative variables, the conversion rate was significantly lower in the $\mathrm{Si}$ RDP-group and Xi-RDP-group: $1 / 35$ case $(2.9 \%)$ and $0 / 35$ cases $(0 \%)$, respectively, versus $5 / 35$ cases $(14.3 \%)$ in the LDP-group $(p=0.045)$. The spleen preservation rate was higher in the RDP-groups, even if it did not reach a statistical

Table 7 Perioperative data

\begin{tabular}{lllll}
\hline & LPD-group & Si-RDP-group & Xi-RDP-group & $p$ value \\
\hline Operative time (min), mean \pm SD & $247 \pm 91$ & $262 \pm 87$ & $225 \pm 59$ & 0.155 \\
Type of operation, $n(\%)$ & & & & 0.027 \\
Distal pancreatectomy & $10(28.6)$ & $21(60)$ & $14(40)$ & \\
Distal splenopancreatectomy & $25(71.4)$ & $14(40)$ & $21(60.0)$ & \\
Pancreas transection, $n(\%)$ & & & & 0.321 \\
Hand-sewn & $8(25.3)$ & $13(38.2)$ & $8(24.2)$ & \\
Stapler & $26(76.5)$ & $21(61.8)$ & $25(75.8)$ & \\
Conversion, $n(\%)$ & $5(14.3)$ & $1(2.9)$ & 0 & 0.045 \\
Hand-assisted laparoscopic surgery & $1(2.9)$ & $1(2.9)$ & 0 & \\
Open surgery & $4(11.4)$ & 0 & 0 & 0.171 \\
Spleen preservation rate, $n(\%)$ & $9 / 20(45 \%)$ & $21 / 29(72.4 \%)$ & $14 / 20(70 \%)$ & 0.710 \\
Overall complications, $n(\%)$ & $17(48.6)$ & $20(57.1)$ & $17(48.6)$ & 0.747 \\
POPF, $n(\%)$ & $14(40.0)$ & $12(34.5)$ & $11(31.4)$ & \\
BL & $11(31.4)$ & $10(28.6)$ & $5(14.3)$ & \\
Grade B & $3(8.6)$ & $2(5.7)$ & $6(17.1)$ & \\
Grade C & 0 & 0 & 0 & \\
Abdominal collection, $n(\%)$ & $3(8.6 \%)$ & $2(5.7 \%)$ & $4(11.4 \%)$ & 0.694 \\
Clavien-Dindo score $\geq$ III, $n(\%)$ & $2(5.7)$ & $1(2.9)$ & $3(8.6)$ & 0.588 \\
Reoperation, $n(\%)$ & $0(0 \%)$ & $0(0 \%)$ & $0(0 \%)$ & 1 \\
Length of hospital stay, mean \pm SD (days) & $11.1 \pm 6.9$ & $9.5 \pm 5.9$ & $9.3 \pm 4.9$ & 0.424 \\
ICU recovery, $n(\%)$ & $5(14.3)$ & $2(5.7)$ & $3(8.6)$ & 0.461 \\
In hospital mortality, $n(\%)$ & $0(0 \%)$ & $1(2.9 \%)$ & $0(0 \%)$ & 1 \\
\hline
\end{tabular}

$P O P F$ post-operative pancreatic fistula, $B L$ biochemical leak, $I C U$ intensive care unit 
significance (72.4\% in the Si-RDP-group and $70 \%$ in the Xi-RDP-group vs $45 \%$ in the LDP-group, $p=0.171$ ). In the RDP subgroups, the number of robotic instruments used was 4 in case of hand sewn closure of the pancreatic stump or 3 in case of the section of the pancreas with a stapler, with a mean number of robotic instruments used for each RDP of 3.3. No differences were reported in terms of overall post-operative complications rate between the three groups: $48.6 \%$ in the LDP-group, $57.1 \%$ in the Si-RDP-group and $48.6 \%$ in the Xi-RDP-group $(p=0.710)$. The incidence of complications with Clavien-Dindo $\geq$ III was similar between the three groups: $5.7 \%$ in the LDP-group, $2.9 \%$ in the SiRDP-group and $8.6 \%$ in the Xi-RDP-group ( $p=0.588$ ). The length of hospital stay was nearly 1 day shorter in the RDP groups than the LDP group even if not statistically significant different: $11.1 \pm 6.9$ days in the LDP-group versus $9.5 \pm 5.9$ days in the Si-RDP-group and $9.3 \pm 4.9$ days in the Xi-RDP-group ( $p=0.424)$. In the LDP-group, the length of hospital stay of converted cases was significantly longer than the cases treated with a minimally invasive approach: $15.6 \pm 10.6$ versus $9.8 \pm 5.7$ days, respectively $(p=0.039)$.

Analyzing the pathological data, no differences were detected between the pathological diagnoses (Table 8). A significant difference was reported in the mean number of harvested lymph nodes, with a lower number reported in the LDP-group: $10.6 \pm 8.1$ vs $14.2 \pm 13.7$ in the Si-RDP-group and $23.2 \pm 16.7$ in the Xi-RDP-group ( $p=0.001)$.

The results of the costs' analysis are summarized in Table 9. No differences were reported about the personnel costs and the hospital stay costs between the three groups

Table 8 Pathological data

\begin{tabular}{lllll}
\hline & LPD-group & Si-RDP-group & Xi-RDP-group & $p$ value \\
\hline Pathological diagnosis, $n(\%)$ & & & & 0.065 \\
PDAC & $8(22.9)$ & $2(5.7)$ & $9(25.7)$ & \\
Adenocarcinoma & 0 & $3(8.6)$ & $1(2.9)$ & \\
IPMN & $2(5.7)$ & $6(17.1)$ & $2(5.7)$ & \\
MCN & $4(11.4)$ & $6(17.1)$ & $1(2.9)$ & \\
SCN & $8(22.9)$ & $4(11.4)$ & $3(8.6)$ & \\
NET & $9(25.7)$ & $10(28.6)$ & $9(25.7)$ & \\
SPT & $1(2.9)$ & 0 & $4(11.4)$ & \\
RCC metastasis & 0 & 0 & $1(2.9)$ & \\
Other & $3(8.6)$ & $4(11.4)$ & $5(14.3)$ & \\
Tumor size (mm), mean \pm SD & $39.3 \pm 23.8$ & $28.4 \pm 19.6$ & $33.1 \pm 12.4$ & 0.074 \\
Harvest lymph nodes, mean \pm SD & $10.6 \pm 8.1$ & $14.2 \pm 13.7$ & $23.2 \pm 16.7$ & 0.001 \\
\hline
\end{tabular}

PDAC pancreatic ductal adenocarcinoma, IPMN intraductal papillary mucinous neoplasm, $M C N$ mucinous cystic neoplasm, $S C N$ serous cystic neoplasm, $N E T$ neuroendocrine tumor, $S P T$ solid pseudopapillary tumor, $R C C$ renal cell carcinoma

Table 9 Costs' analysis

\begin{tabular}{|c|c|c|c|c|c|}
\hline & LPD-group & Si-RDP-group & Xi-RDP-group & $p$ value & $p$ value \\
\hline $\begin{array}{l}\text { Personnel's costs }(€) \text {, median } \\
\text { [Q1-Q3] }\end{array}$ & $1143[957-1385]$ & $1234[1108-1612]$ & 1183 [907-1360] & 0.132 & \\
\hline $\begin{array}{l}\text { Hospital stay costs }(€) \text {, median } \\
\text { [Q1-Q3] }\end{array}$ & $4200[2940-5460]$ & $2940[2520-4200]$ & $3360[2520-4620]$ & 0.069 & \\
\hline $\begin{array}{l}\text { Consumables costs }(€) \text {, median } \\
\text { [Q1-Q3] }\end{array}$ & $1505[1151-1772]$ & $3434[3061-3646]$ & 3409 [3201-3579] & $<0.001$ & $\begin{array}{l}<0.05 \text { LDP-group vs Si-RDP- } \\
\text { group } \\
<0.05 \text { LDP-group vs Xi-RDP- } \\
\text { group }\end{array}$ \\
\hline $\begin{array}{l}\text { Overall variable costs }(€) \text {, median } \\
\text { [Q1-Q3] }\end{array}$ & $6968[5961-8392]$ & 7,856 [7117-9754] & 7981 [7095-9040] & 0.016 & $\begin{array}{l}<0.05 \text { LDP-group vs Si-RDP- } \\
\text { group } \\
<0.05 \text { LDP-group vs Xi-RDP- } \\
\text { group }\end{array}$ \\
\hline Overall costs $(€)$, median [Q1-Q3] & $7002[5996-8426]$ & $9440[8700-11338]$ & 9809 [8922-10867] & $<0.001$ & $\begin{array}{l}<0.05 \text { LDP-group vs Si-RDP- } \\
\text { group } \\
<0.05 \text { LDP-group vs Xi-RDP- } \\
\text { group }\end{array}$ \\
\hline
\end{tabular}


( $p$-value $=0.132$ and $p=0.069$, respectively). The comparison of the consumable costs showed significant higher costs of the Si-RDP-group and the Xi-RDP-group respect to the LPD-group (median values being $€ 3434$ and $€ 3409$ versus $€ 1505$, respectively, $p<0.05)$. Overall variable costs and overall costs including fixed costs of the Si-RDP-group and the Xi-RDP-group resulted significantly higher respect to the LPD group: median values being $€ 7856$ and $€ 7981$ versus $€ 6968$, and $€ 9440$ and $€ 9809$ versus $€ 7002$, respectively $(p<0.05)$. However, at multivariate analysis, adjusting for age, ASA risk score and spleen preservation, overall variable costs were no more significantly different between the

Table 10 Univariate analysis

\begin{tabular}{lcc}
\hline & Coef (Std.Err) & $p$ value \\
\hline Overall variable costs & $0.003(0.002)$ & 0.116 \\
Age & $0.01(0.06)$ & 0.855 \\
Male gender & $0.01(0.01)$ & 0.107 \\
Body mass index & $0.16(0.05)$ & 0.002 \\
ASA risk score & $-0.04(0.07)$ & 0.543 \\
Benign tumor & $-0.15(0.06)$ & 0.012 \\
Spleen preservation & $-0.07(0.13)$ & 0.628 \\
Conversion & & \\
Overall costs & $0.002(0.002)$ & 0.264 \\
Age & $0.002(0.06)$ & 0.97 \\
Male gender & $0.01(0.01)$ & 0.135 \\
Body mass index & $0.15(0.05)$ & 0.003 \\
ASA risk score & $-0.05(0.06)$ & 0.422 \\
Benign tumor & $-0.10(0.06)$ & 0.086 \\
Spleen preservation & $-0.16(0.13)$ & 0.205 \\
Conversion &
\end{tabular}

LPD-group and the Xi-RPD-group ( $p=0.099)$ (Tables 10 and 11). Moreover, when distinguishing for the intention to spleen preservation on the basis of the preoperative diagnosis, the multivariate analysis revealed that the overall variable costs were no longer significantly different between the LPD-group versus the Si-RPD-group and the Xi-RPD-group in the subgroup of patients in which the spleen preservation was indicated ( $p=0.115$ and $p=0.261$, respectively) (Table 11).

\section{Discussion}

The economic impact of the robotic approach on pancreatic surgery, and particularly on distal pancreatectomy, is still under debate, with controversial published studies [3, 9-14]. Indeed, although the majority of authors reported higher costs associated to RAS with respect to DML [11, 24], some evidences underlining the limitations and biases of the majority of the cost-analysis published so far, have opened new perspectives on this specific issue [6, 15].

Therefore, as from a clinical point of view RDP seems to be associated with some positive impact on the outcomes with respect to the LDP, it is still a matter of debate whether the use of the da Vinci is sustainable, and whether the advantages of the da Vinci Surgical System can justify its use for distal pancreatectomy.

For these reasons, to evaluate the costs-effectiveness of RAS and DML in this setting, different factors to be evaluated comprise both those strictly related to the instrumentation and its use and those referring to the clinical outcomes. Thus, to perform our structured cost-analysis, we divided the costs in fixed and variable costs, including

Table 11 Multivariate analysis

\begin{tabular}{|c|c|c|c|c|}
\hline & & \multicolumn{2}{|l|}{ Coef (Std.Err) } & $p$ value \\
\hline \multicolumn{5}{|l|}{ Overall variable costs } \\
\hline LDP-group vs Si-RDP-group & \multicolumn{2}{|r|}{$0.18(0.07)$} & & 0.016 \\
\hline LDP-group vs Xi-RDP-group & \multicolumn{2}{|r|}{$0.11(0.07)$} & & 0.099 \\
\hline \multicolumn{5}{|l|}{ Overall costs } \\
\hline LDP-group vs Si-RDP-group & \multicolumn{2}{|r|}{$0.33(0.06)$} & & $<0.001$ \\
\hline LDP-group vs Xi-RDP-group & \multicolumn{2}{|r|}{$0.31(0.06)$} & & $<0.001$ \\
\hline & \multicolumn{2}{|c|}{ No intention spleen preservation } & \multicolumn{2}{|c|}{ Intention spleen preservation } \\
\hline & Coef (Std.Err) & $p$ value & Coef (Std.Err) & $p$ value \\
\hline \multicolumn{5}{|l|}{ Overall variable costs } \\
\hline LDP-group vs Si-RDP-group & $0.25(0.13)$ & 0.058 & $0.14(0.09)$ & 0.115 \\
\hline LDP-group vs Xi-RDP-group & $0.20(0.09)$ & 0.037 & $0.11(0.10)$ & 0.261 \\
\hline \multicolumn{5}{|l|}{ Overall costs } \\
\hline LDP-group vs Si-RDP-group & $0.40(0.12)$ & 0.001 & $0.30(0.08)$ & $<0.001$ \\
\hline LDP-group vs Xi-RDP-group & $0.39(0.08)$ & $<0.001$ & $0.30(0.09)$ & 0.001 \\
\hline
\end{tabular}


in the first the purchase and maintenance of the technology, and in the latter the disposable instruments (consumable costs), the length of stay and the post-operative course (hospital stay costs), and the operating room personnel costs (personnel costs) which are associated to the OT.

Interestingly, despite our costs' analysis confirmed that the overall cost including fixed costs and overall variable costs of RDP resulted higher compared with that of the LDP, at univariate and multivariate analysis, the overall variable costs were no longer significantly different between the LPD-group and the Xi-RPD-group. In our opinion, this data can be explained by the standardization of the technique, the perioperative outcomes, and by a discount of robotic instruments' purchase costs coming from the multidisciplinary high-volume center setting as well.

In fact, the standardization of the technique with the improvement in robotic expertise, allows to optimize the use of instruments, by performing all phases of the surgical operation with an average of only 3.3 robotic instruments, therefore contributing to minimize the CCs [13]. Indeed, in our series we used a basic set for each surgical approach including the essential instruments to perform the operation, and all other instruments were used on request, only if strictly necessary. In detail, in the robotic approach, we standardized the use of the monopolar scissors on the right hand and of the Maryland bipolar forceps in the left hand for dissection, and of the Cadiere grasper for retraction in the fourth arm, while a fourth instrument was used only in selected cases when suturing was needed. Instead, by laparoscopy we always used an energy device for dissection.

Among the perioperative outcomes, OT, conversion rate, and LOS are the main key factors that might have had a major impact on costs. Indeed, in our series, the reported shorter mean operative time of the Xi-RDP-group with respect to Si-RDP-group and LDP-group, even if not statistically significant, has played a role in reducing the PCs, in line with similar works already reported also for other indications [15]. In fact, the reduced docking time of the da Vinci Xi with respect to the da Vinci Si, as well as the improvement of the surgical workflow, represent two well consolidated advantages of the latest version of the da Vinci System, that can independently influence the OT and therefore, indirectly, also the costs. In our series, several factors might contribute to explain the reduction of operative time and PCs of Xi-RDP also in comparison with LDP, firstly the higher experience with RAS [15], and secondly the advantages of RAS over DML that simplify the execution of all the difficult tasks of LDP, such as dissection of the pancreatic body and tail from splenic vessels, suturing, control of bleeding, completion of the retropancreatic tunnel, and suturing the pancreatic stump with the closure of Wirsung duct [25].
Other important perioperative factors influencing costs in our series, as well as in previous papers [6, 24, 26-30], are the conversion rate and the LOS. In particular, the significantly lower conversion rate of RDP that we reported, index assessing the ability to deal with the complexity of the procedures and complete them with a minimally invasive approach thanks to the greater dexterity and precision of robotic manipulation in vessel dissection and bleeding control [29], could partially justify also the shorter LOS in the RDP groups, that resulted nearly 1 day shorter than the LDP group. In fact, the LOS of patients that had undergone LDP completed with the laparoscopic approach was significantly lower than the LOS of converted cases of LDP-groups, while it was similar to the LOS of RDP groups.

In this respect, it has also to be noticed that, despite the minimally invasive approach and the relatively low incidence of complications, we reported a mean LOS longer as compared with other robotic and laparoscopic case series of international literature. However, our data are comparable with the analysis conducted in an Italian multicenter cohort of 236 patients that had undergone RDP [31], and this could probably be due to cultural factors with a more cautious policy used for discharging patients from hospital, as in Italy patients expect to leave hospital only when fully recovered and therefore needing little outpatient [32]. As a result, these data suggest that the possible optimization of costs could theoretically be even greater.

Another aspect which deserves to be highlighted among the positive impact of the RAS versus the DML on the outcomes of the distal pancreatectomy, although difficult to quantify within a cost analysis, is the higher spleen preservation rate. In this regard, our data are in line with literature $[2,7,17,33]$, as the greater effectiveness of robotic system in controlling splenic vessels bleeding resulted in a higher spleen preservation rate associated with RDP among patients scheduled for spleen preservation with respect to LDP. This outcome is important not only for the biological benefit of spleen preservation, but also as the reduced use of stapler and stapler charges, necessary for the section of the splenic vessel in case of splenectomy, could contribute to reduce the CCs of RDP.

Interestingly, in this regard, the multivariate analysis of our costs' analysis revealed that the overall variable costs were no longer significantly different between LPD and RPD, both for the da Vinci Si and the da Vinci Xi, in the subgroup of patients in which the spleen preservation was indicated on the basis of the preoperative diagnosis. These data suggest that the use of the da Vinci Surgical System, both $\mathrm{Si}$ and $\mathrm{Xi}$, could be particularly indicated in those cases in which the spleen preservation is scheduled.

From a histological standpoint, we reported a higher percentage of cystic lesions in the Si-RDP-group, while on the contrary a higher percentage of pancreatic carcinoma was 
reported in the Xi-RDP-group, explaining also the higher number of lymph nodes harvest in the Xi-RDP-group. In fact, while in the initial experience with RAS we preferred to treat benign lesions, with the overcoming of the learning phase with the robotic technology, more and more complex cases were treated with RDP, particularly since the introduction of the da Vinci Xi. We acknowledge that this aspect could introduce a bias, but it is interesting to note that although the complexity of cases has increased over time within the RDP whole group, the outcomes of patients belonging to the Xi-RDP-group were not worse respect to those of the Si-RDP-group and, in some cases, resulted even better. Furthermore, although the number of pancreatic malignant tumors was similar between LDP-group and $\mathrm{Xi}$-RDP-group, the number of harvested lymph nodes was significantly higher in the latter, probably as a consequence that, because of increasing confidence with the robotic system acquired over time, as well as the improvement offered by the $\mathrm{Xi}$ version, more complex cases of malignant tumors located in the isthmus or central part of the body of the pancreas needing a more extended lymphadenectomy, were treated with a minimally invasive technique. This is a crucial aspect, to encourage the use of robot, particularly if included in a setting of possible optimization of costs. Indeed, even though the use of robotic technology to perform a robotic DP was first reported by Melvin in 2003 [34], so far the use of minimally invasive technique is limited to highly specialized centers, while the open approach is still chosen in many surgical center. Actually, the technical advantages of robotic systems could allow the expansion of the adoption of minimally invasive surgery in the field of pancreatic surgery [35], without impairing the clinical outcomes, and, therefore, on the cost-effective management of patients that require a DP, the higher possibility to offer a minimally invasive approach also for malignant and more complex tumors might be increasingly taken in consideration. This could be particularly important also for the LOS reduction and the faster return to normal daily activities, making more likely a shorter time between surgery and adjuvant chemotherapy compared to an open DP [36]. Although this data can be difficult to be evaluated in economic terms, we think that it should be taken into consideration when analyzing the right balance between these advantages and the higher costs of RDP.

Our study has some limitations. First of all, its retrospective nature, the heterogeneity of the sample and the not standardized choice of the laparoscopic versus robotic approach. However, using a case-control matching analysis we tried to mitigate these issues by obtaining three comparable groups. Another criticism might be the exclusion of robotic staplers' costs to avoid bias in the analysis of consumable costs. Indeed, as previously declared, at the time our $\mathrm{Si}$ interventions were performed, they were not available for that robotic platform. Furthermore, so far there is no evidence in literature of the superiority of the endo-wristed staplers respect to the laparoscopic ones, and the use of anyone of them during an RDP is merely a surgeon's choice. Therefore, considering the costs of endo-wristed staplers during the analysis would have introduced a bias. For this reason, in order to have three homogeneous groups, when the robotic staplers were used in the Xi-RDP-group we considered the costs of the corresponding laparoscopic staplers and charges, as they were always usable in all cases. Moreover, since this study comes from a high-volume center for pancreatic surgery and also for RAS, the results of the analysis may not be applicable to all centers, also because the cost-analysis was performed using specific resources use and economic data of the accounting department of our hospital and it could be difficult to generalize these data to other parts of the world. In this regard, although currently difficult to define a threshold, the use of the da Vinci Surgical System in a highvolume RAS center, can contribute itself to the reduction of the fixed costs, by reducing the depreciation charge, and of each operation OVCs of RDP thanks to reduction of the CCs. Indeed, in our experience, thanks to the high number of robotic operations performed each year at the Multidisciplinary Center of Robotic Surgery at Cisanello Hospital in Pisa, we obtained a $2 \%$ discount of robotic instruments' purchase costs, partially contributing to reduce the gap of CCs between the LDP and RDP. Moreover, performing even more than a thousand robotic interventions per year, as we have experienced at our Centre, thanks to the purchase of a large number of robotic instruments it is possible to obtain a further bulk discount, thus reducing the average cost of robotic instruments for each robotic intervention, with the possibility to obtain a further reduction of CCs of RDP. This represents another reason, in addition to those mentioned above, for further encourage the wide spreading of robotic technologies in the field of pancreatic surgery. Therefore, it may well be crucial to encourage the spread of multidisciplinary high-volume centers, in which surgeons coming from different hospitals can have access with the opportunity to operate on their own patients, benefiting from the most advanced technologies and also reducing costs at the same time. This could be one of the key points to make sustainable the use of robots for surgical operation, particularly for more complex cases, such as those involving pancreatic surgery. Furthermore, of note that according to accounting standards fixed costs attributable to the single intervention were estimated considering the 5-year amortization period of the instruments. This period is obviously limited when compared to the actual period of use of the technologies, thus spreading costs over the entire product life cycle can give a more realistic picture of the economic impact and in this case the gap in fixed costs between LDP and RDP would be reduced. Finally, this study is a cost analysis and further 
studies properly evaluating the cost-effectiveness of RDP, also including an assessment of the quality of life and the impact of the procedure on long-term outcomes, would shed light on the cost-effectiveness of RDP vs LDP.

In conclusion, RAS is more expensive than DML for DP because of higher acquisition and maintenance costs. The flattening of these differences considering only the variable costs suggests a possible optimization of the cost-effectives of RAS, particularly in high-volume centers. As a result, the advantages of robotic technology could be encouraged in this setting, as it can favor the use of minimally invasive approach for DP by a larger cohort of pancreatic surgeons and expand the field of this approach to more complex pancreatic lesions, so far treated by open surgery. Moreover, RAS might be particularly indicated for MIDP when the spleen preservation is scheduled.

Acknowledgements The authors thank the Arpa Foundation for the support.

Funding Open Access funding provided by Università di Pisa.

\section{Compliance with ethical standards}

Disclosures Dr. Di Franco, Dr. Peri, Dr. Lorenzoni, Dr. Palmeri, Dr. Furbetta, Dr. Guadagni, Dr. Gianardi, Dr. Bianchini, Dr. Pollina, Prof. Melfi, Dr. Mamone, Dr. Milli, Prof. Di Candio, Prof. Turchetti, Prof. Pietrabissa, Prof. Morelli have no conflict of interest or financial ties to disclose.

Open Access This article is licensed under a Creative Commons Attribution 4.0 International License, which permits use, sharing, adaptation, distribution and reproduction in any medium or format, as long as you give appropriate credit to the original author(s) and the source, provide a link to the Creative Commons licence, and indicate if changes were made. The images or other third party material in this article are included in the article's Creative Commons licence, unless indicated otherwise in a credit line to the material. If material is not included in the article's Creative Commons licence and your intended use is not permitted by statutory regulation or exceeds the permitted use, you will need to obtain permission directly from the copyright holder. To view a copy of this licence, visit http://creativecommons.org/licenses/by/4.0/.

\section{References}

1. Cuschieri A (1994) Laparoscopic surgery of the pancreas. J R Coll Surg Edinb 39:178-184

2. Asbun HJ, Moekotte AL, Vissers FL, Kunzler F, Cipriani F, Alseidi A et al (2020) The Miami international evidence-based guidelines on minimally invasive pancreas resection. Ann Surg 271:1-14. https://doi.org/10.1097/SLA.0000000000003590

3. Venkat R, Edil BH, Schulick RD, Lidor AO, Makary MA, Wolfgang CL (2012) Laparoscopic distal pancreatectomy is associated with significantly less overall morbidity compared to the open technique: a systematic review and meta-analysis. Ann Surg 255:1048-1059. https://doi.org/10.1097/SLA.0b013e318251ee09
4. Shin SH, Kim SC, Song KB, Hwang DW, Lee JH, Lee D et al (2015) A comparative study of laparoscopic vs open distal pancreatectomy for left-sided ductal adenocarcinoma: a propensity score-matched analysis. J Am Coll Surg 220:177-185. https://doi. org/10.1016/j.jamcollsurg.2014.10.014

5. Sharpe SM, Talamonti MS, Wang E, Bentrem DJ, Roggin KK, Prinz RA et al (2015) The laparoscopic approach to distal pancreatectomy for ductal adenocarcinoma results in shorter lengths of stay without compromising oncologic outcomes. Am J Surg 209:557-563. https://doi.org/10.1016/j.amjsurg.2014.11.001

6. Hu YH, Qin YF, Yu DD, Li X, Zhao YM, Kong DJ et al (2020) Meta-analysis of short-term outcomes comparing robot-assisted and laparoscopic distal pancreatectomy. J Comp Eff Res 9:201218. https://doi.org/10.2217/cer-2019-0124

7. Yang SJ, Hwang HK, Kang CM, Lee WJ (2020) Revisiting the potential advantage of robotic surgical system in spleen-preserving distal pancreatectomy over conventional laparoscopic approach. Ann Transl Med 8:188-188. https://doi.org/10.21037 /atm.2020.01.80

8. Turchetti G, Palla I, Pierotti F, Cuschieri A (2012) Economic evaluation of da Vinci-assisted robotic surgery: a systematic review [Internet]. Surg Endosc 26:598-606

9. Butturini G, Damoli I, Crepaz L, Malleo G, Marchegiani G, Daskalaki D et al (2015) A prospective non-randomised single-center study comparing laparoscopic and robotic distal pancreatectomy. Surg Endosc 1(29):3163-3170. https://doi. org/10.1007/s00464-014-4043-3

10. Waters JA, Canal DF, Wiebke EA, Dumas RP, Beane JD, Aguilar-Saavedra JR et al (2010) Robotic distal pancreatectomy: cost effective? Surgery 148:814-823. https://doi.org/10.1016/j. surg.2010.07.027

11. Souche R, Herrero A, Bourel G, Chauvat J, Pirlet I, Guillon F et al (2018) Robotic versus laparoscopic distal pancreatectomy: a French prospective single-center experience and costeffectiveness analysis. Surg Endosc 32:3562-3569. https://doi. org/10.1007/s00464-018-6080-9

12. Turchetti G, Pierotti F, Palla I, Manetti S, Freschi C, Ferrari V et al (2017) Comparative health technology assessment of robotic-assisted, direct manual laparoscopic and open surgery: a prospective study. Surg Endosc [internet] 31:543-551. https ://doi.org/10.1007/s00464-016-4991-x

13. Vicente E, Núñez-Alfonsel J, Ielpo B, Ferri V, Caruso R, Duran $\mathrm{H}$ et al (2020) A cost-effectiveness analysis of robotic versus laparoscopic distal pancreatectomy. Int J Med Robot Comput Assist Surg. https://doi.org/10.1002/rcs.2080

14. Alfieri S, Butturini G, Boggi U, Pietrabissa A, Morelli L, Vistoli F et al (2019) Short-term and long-term outcomes after robotassisted versus laparoscopic distal pancreatectomy for pancreatic neuroendocrine tumors ( $\mathrm{pNETs}$ ): a multicenter comparative study. Langenbeck's Arch Surg. https://doi.org/10.1007/s0042 3-019-01786-x

15. Morelli L, Di Franco G, Lorenzoni V, Guadagni S, Palmeri M, Furbetta N et al (2019) Structured cost analysis of robotic TME resection for rectal cancer: a comparison between the da Vinci Si and Xi in a single surgeon's experience. Surg Endosc 33:1858-1869. https://doi.org/10.1007/s00464-018-6465-9

16. De Pastena M, Esposito A, Paiella S, Surci N, Montagnini G, Marchegiani G et al (2020) Cost-effectiveness and quality of life analysis of laparoscopic and robotic distal pancreatectomy : a propensity score-matched study. Surg Endosc. https://doi. org/10.1007/s00464-020-07528-1

17. Morelli L, Guadagni S, Palmeri M, Di Franco G, Caprili G, D'Isidoro C et al (2016) A case-control comparison of surgical and functional outcomes of robotic-assisted spleen-preserving left side pancreatectomy versus pure laparoscopy. J Pancreas $17: 241-246$ 
18. Kimura W, Yano M, Sugawara S, Okazaki S, Sato T, Moriya $\mathrm{T}$ et al (2010) Spleen-preserving distal pancreatectomy with conservation of the splenic artery and vein: techniques and its significance. J Hepatobiliary Pancreat Sci 17:813-823. https:// doi.org/10.1007/s00534-009-0250-z

19. Warshaw AL (1988) Conservation of the spleen with distal pancreatectomy. Arch Surg 123:550-553. https://doi.org/10.1001/ archsurg.1988.01400290032004

20. Dindo D, Demartines N, Clavien P-A (2004) Classification of surgical complications: a new proposal with evaluation in a cohort of 6336 patients and results of a survey. Ann Surg 240:205-213. https://doi.org/10.1097/01.sla.0000133083.54934 .ae

21. Wente MN, Veit JA, Bassi C, Dervenis C, Fingerhut A, Gouma DJ et al (2007) Postpancreatectomy hemorrhage (PPH)-an international study group of pancreatic surgery (ISGPS) definition. Surgery 142:20-25. https://doi.org/10.1016/j.surg.2007.02.001

22. Wente MN, Bassi C, Dervenis C, Fingerhut A, Gouma DJ, Izbicki JR et al (2007) Delayed gastric emptying (DGE) after pancreatic surgery: a suggested definition by the international study group of pancreatic surgery (ISGPS). Surgery 142:761-768. https://doi. org/10.1016/j.surg.2007.05.005

23. Bassi C, Marchegiani G, Dervenis C, Sarr M, Abu Hilal M, Adham $\mathrm{M}$ et al (2017) The 2016 update of the international study group (ISGPS) definition and grading of postoperative pancreatic fistula: 11 years after. Surgery 161:584-591. https://doi. org/10.1016/j.surg.2016.11.014

24. Xu SB, Jia CK, Wang JR, Zhang RC, Mou YP (2019) Do patients benefit more from robot assisted approach than conventional laparoscopic distal pancreatectomy? a meta-analysis of perioperative and economic outcomes. J Formos Med Assoc 118:268-278. https ://doi.org/10.1016/j.jfma.2018.05.003

25. Morelli L, Di Franco G, Guadagni S, Palmeri M, Furbetta N, Funel N et al (2018) Robotic-assisted versus open left pancreatectomy for cystic tumours: a single-centre experience. J Minim Access Surg. https://doi.org/10.4103/jmas.JMAS_158_18

26. Magge DR, Zenati MS, Hamad A, Rieser C, Zureikat AH, Zeh HJ et al (2018) Comprehensive comparative analysis of cost-effectiveness and perioperative outcomes between open, laparoscopic, and robotic distal pancreatectomy. Hpb 20:1172-1180. https://doi. org/10.1016/j.hpb.2018.05.014

27. Zhou J-Y, Xin C, Mou Y-P, Xu X-W, Zhang M-Z, Zhou Y-C et al (2016) Robotic versus laparoscopic distal pancreatectomy: a metaanalysis of short-term outcomes. PLoS ONE 11:e0151189. https ://doi.org/10.1371/journal.pone.0151189

28. Chen S, Zhan Q, Chen JZ, Jin JB, Deng XX, Chen H et al (2015) Robotic approach improves spleen-preserving rate and shortens postoperative hospital stay of laparoscopic distal pancreatectomy: a matched cohort study. Surg. Endosc. 29:3507-3518. https://doi. org/10.1007/s00464-015-4101-5

29. Liu R, Liu Q, Zhao ZM, Tan XL, Gao YX, Zhao GD (2017) Robotic versus laparoscopic distal pancreatectomy: a propensity score-matched study. J Surg Oncol 116:461-469. https://doi. org $/ 10.1002 /$ jso. 24676

30. Guerrini GP, Lauretta A, Belluco C, Olivieri M, Forlin M, Basso $S$ et al (2017) Robotic versus laparoscopic distal pancreatectomy: an up-to-date meta-analysis. BMC Surg. https://doi.org/10.1186/ s12893-017-0301-3

31. Alfieri S, Boggi U, Butturini G, Pietrabissa A, Morelli L, Di Sebastiano P et al (2020) Full robotic distal pancreatectomy: safety and feasibility analysis of a multicenter cohort of 236 patients. Surg Innov 27:11-18. https://doi.org/10.1177/15533 50619868112

32. Giulianotti PC, Coratti A, Angelini M, Sbrana F, Cecconi S, Balestracci $\mathrm{T}$, et al. Robotics in general surgery: personal experience in a large community hospital. Arch. Surg. [Internet] 2003 1 [cited 2017 29];138:777-84. http://archsurg.jamanetwork.com/ article.aspx?. https://doi.org/10.1001/archsurg.138.7.777

33. Choi SH, Seo MA, Hwang HK, Kang CM, Lee WJ (2012) Is it worthwhile to preserve adult spleen in laparoscopic distal pancreatectomy? Perioperative and patient-reported outcome analysis. Surg Endosc 26:3149-3156. https://doi.org/10.1007/s0046 4-012-2306-4

34. Melvin WS, Needleman BJ, Krause KR, Ellison EC (2003) Robotic resection of pancreatic neuroendocrine tumor. J Laparoendosc Adv Surg Tech 13:33-36. https://doi.org/10.1089/10926 4203321235449

35. Di Benedetto F, Ballarin R, Magistri P (2019) Robotic distal pancreatectomy: can results overcome cost-effectiveness prejudices? HepatoBiliary Surg Nutr 8:304-306. https://doi.org/10.21037/ hbsn.2019.01.23

36. van Hilst J, Korrel M, de Rooij T, Lof S, Busch OR, Groot Koerkamp B et al (2019) Oncologic outcomes of minimally invasive versus open distal pancreatectomy for pancreatic ductal adenocarcinoma: a systematic review and meta-analysis. Eur J Surg Oncol 45:719-727. https://doi.org/10.1016/j.ejso.2018.12.003

Publisher's Note Springer Nature remains neutral with regard to jurisdictional claims in published maps and institutional affiliations. 